\title{
Radial Drawings of Graphs: Geometric Constraints and Trade-Offs ${ }^{\star}$
}

\author{
Emilio Di Giacomo, Walter Didimo, and Giuseppe Liotta \\ Dip. Ingegneria Elettronica e dell'Informazione - Università degli Studi di Perugia \\ \{digiacomo, liotta, didimo\}@diei.unipg.it
}

\begin{abstract}
This paper studies how to compute radial drawings of graphs by taking into account additional geometric constraints which correspond to typical aesthetic and semantic requirements for the visualization. The following requirements are considered: vertex centrality, edge crossings, curve complexity, and vertex radial distribution. Trade-offs among these requirements and efficient drawing algorithms are presented.
\end{abstract}

\section{Introduction}

The readability of a drawing of a graph describes its effectiveness in conveying the information associated with the graph itself. The set of geometric requirements related to the readability of a drawing are called aesthetic requirements and those related to the semantics are called semantic requirements (see, e.g., 6 6 11 18]). While aesthetic requirements are usually expressed as geometric optimization goals for a graph drawing algorithm, semantic requirements express constraints that must be satisfied in the output visualization and are provided to the algorithm as an additional input. Taking into account more than one aesthetic requirement typically translates into a multi-objective optimization problem, which is inherently characterized by trade-offs. Many such trade-offs have received attention in the literature, including area vs. angular resolution, area vs. aspect ratio, edge crossings vs. number of bends (see also 6 17]).

This paper is devoted to the study of aesthetic and semantic requirements which occur when computing radial drawings of graphs. A radial drawing of a graph is such that every vertex is drawn on one of $k$ concentric circles and the edges are polygonal chains. Radial drawings arise in all those applications where it is important to display a graph with the constraint that some vertices are drawn "more central" than others. Examples of such applications include social networks analysis (visualization of policy networks and co-citation graphs), cybergeography (visualization of Web maps and communities), and bioinformatics (visualization of protein-protein interaction diagrams). See, e.g., 910.

In spite of their importance in practice, the study of visualization algorithms and systems that compute radial drawings of graphs by taking into account different aesthetic and semantic requirements has not yet received enough attention. Namely, there exist visualization systems that compute radial drawings

\footnotetext{
^ Research partially supported by MIUR under Project "ALGO-NEXT".
} 
that satisfy the semantic requirement of placing each vertex on a given circle corresponding to its centrality 34; however, these systems often disregard some basic aesthetic requirements, for example they give rise to drawings with several edge crossings. On the other hand, the problem of computing radial drawings of planar graphs with no edge intersections and no bends along the edges has been studied in [7; however, the drawing algorithm does not take into account any semantic requirements. The problem of testing whether a graph admits a crossing-free radial drawing that satisfies the assigned centrality of the vertices and where the edges are monotone Jordan curves has been studied by Bachmaier et al. [12]; however, only a planar embedding (not a drawing) is returned and furthermore the number of bends along the edges is not considered.

We describe different algorithms that compute radial drawings of graphs and present trade-offs among typical aesthetic and semantic requirements. We consider the following requirements: vertex centrality (each vertex should be placed on the circular level corresponding to its centrality), edge crossings (the number of edge crossings should be small), curve complexity (the number of bends along each edge should be small), radial distribution of the vertices (the vertices should be uniformly distributed in a radial fashion on a polar grid [16]). An outline of the main results in this paper is as follows.

- We show that in general it is not possible to compute radial drawings of planar graphs where no two edges cross, the number of bends is zero, the radial distribution of the vertices is uniform and the vertex centrality is satisfied. Since vertex centrality is a semantic requirement and hence it must be satisfied and since reducing the number of crossings is recognized as one of the most important aesthetics in graph drawing applications (see, e.g., [1415]), we give precedence to these two requirements over the others.

- A consequence of a result by Pach and Wenger [13] is that radial drawings of planar graphs having zero edge crossings, uniform radial distribution, and respecting vertex centrality can be computed at the price of a high curve complexity (there can be a linear number of bends per edge). We show how to achieve low curve complexity by describing a linear-time algorithm that computes radial drawings of planar graphs with no edge crossings, uniform radial distribution, and at most three bends per edge.

- Trade-offs between the number of bends per edge and the uniform radial distribution are studied. We describe linear-time algorithms that can further reduce the number of bends at the expenses of a non-uniform radial distribution. We show that every planar graph with assigned vertex centrality admits a radial leveled planar drawing with at most one bend per edge if the radii of the circles are not given as part of the input and with at most two bends per edge if the radii are fixed in advance.

The results in this paper are based on a combination of geometric and graph theoretic techniques. Three bends per edge and uniform radial distribution are achieved by exploiting properties of star-shaped polygons and Hamiltonian augmentation. Radial drawings with at most two bends per edge and with at most 
one bend per edge are computed by using canonical ordering [5] and curve embedding [8], respectively. Figure 1] shows different radial drawings of the same graph with assigned centrality for the vertices: the white vertices are assigned to the external circle, the black ones to the inner circles, and the grey vertices to the mid circle. The drawing of Figure 1(a) has curve complexity zero but has a high number of crossings and poor radial distribution of the vertices. The drawing of Figure 1(b) is optimal in terms of crossings and radial distribution of the vertices and has a small number of bends per edge. The number of bends per edge is reduced in Figure 1(c) at the expenses of a sub-optimal radial distribution.

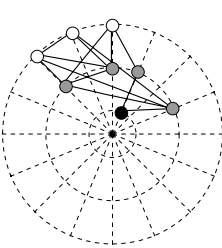

(a)

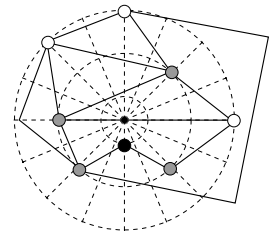

(b)

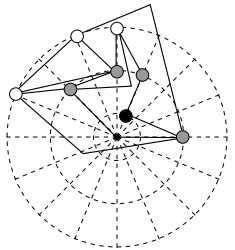

(c)

Fig. 1. Three radial drawings of the same graph with assigned centrality for the vertices. The three drawings present different trade-offs among aesthetic requirements.

\section{Preliminaries}

We assume familiarity with basic concepts of graph drawing [6. Let $G$ be a planar graph and let $\Gamma$ be a drawing of $G$. If the edges of $G$ are represented in $\Gamma$ as a polygonal chain we say that $\Gamma$ is a polyline drawing. The intersection between two consecutive straight-line segments in the polygonal chain representing an edge $e$ is called a bend of $e$. If a drawing $\Gamma$ is such that each edge is represented with a polygonal chain with at most $b$ bends we say that $\Gamma$ is a $b$-bend drawing of $G$. A 0-bend drawing is also called a straight-line drawing. A radial drawing of a graph $G$ is a polyline drawing of $G$ such that each vertex is drawn as a point of one among a set $\mathcal{C}$ of concentric circles. We assume that the center of the circles of $\mathcal{C}$ is the origin $o$ of the Euclidean plane. A ray is a half-line with origin the point $o$. Given a ray $\varrho$ we denote by $\angle \varrho$ the counterclockwise angle required to bring the positive $x$-axis into correspondence with $\varrho$. Given two rays $\varrho_{a}$ and $\varrho_{b}$, we define $\angle \varrho_{a} \varrho_{b}=\angle \varrho_{b}-\angle \varrho_{a}$, where angles are measured modulo $2 \pi$. A star-shaped polygon $\mathcal{P}$ is a polygon in which there exists an interior point $p$ such that all the boundary points of $\mathcal{P}$ are visible from $p$. The set of all points $p$ satisfying this property is called the kernel of $\mathcal{P}$.

\section{Semantic and Aesthetic Requirements}

As anticipated in the introduction, the semantic requirement that we take into account is vertex centrality, that associates the vertices in the input graph 
$G(V, E)$ to radial levels. For each vertex $v \in V$, its centrality (radial level) is specified as part of the input and the algorithm computes a drawing such that $v$ is drawn on a circle corresponding to the given centrality. More formally, a leveled graph $G=(V, E, \phi)$, consists of a set of vertices $V$, a set of edges $E$ and a function $\phi: V \rightarrow\{0,1, \ldots, k-1\}$ that maps each vertex to an integer between 0 and $k-1$, which represents its centrality. A radial leveled drawing of $G=(V, E, \phi)$ is a radial drawing of $G=(V, E, \phi)$ on a set of $k$ concentric circles $\mathcal{C}=\left\{C_{0}, \ldots, C_{k-1}\right\}$ (with the radius of $C_{i}$ greater than the radius of $C_{i+1}$ ) such that each vertex $v \in V$ is drawn as a point of circle $C_{\phi(v)}$. The value $k$ will be also called the level number of $G$, and will be denoted as $\lambda(G)$. Since in this paper we only study radial leveled drawings of leveled graphs, from now on we will often call them simply "radial drawings", omitting the term "leveled".

Concerning the visual appeal of radial drawings, we focus on the following aesthetic requirements:

- CROssings. A crossing between two edges occurs if the two edges share a point different from their end-vertices. A drawing should have as few crossings as possible, ideally 0 if the graph is planar.

- CURVE COMPLEXITY. The curve complexity is the maximum number of bends per edge. A readable drawing typically has low curve complexity (see, e.g., 6]).

- RADIAL DISTRIBUTION. In a radial drawing it is desirable that the vertices are uniformly distributed on a polar grid (see, e.g., [16]). Namely, the difference between the radii of any two consecutive circles should be constant and equal to the radius of the smallest circle; also, the angular distance between any two consecutive vertices encountered with a radial sweep of the drawing should be constant. More formally, we shall measure the radial distribution of the vertices in terms of:

- Radial Distance Ratio (RDR). Denote by $r_{i}$ the radius of $C_{i}(i=$ $0, \ldots, k-1)$ and set $r_{k}=0$. Define $\Delta r_{i}=r_{i}-r_{i+1}(i=0, \ldots, k-1)$, $\Delta r_{\text {min }}=\min _{i}\left\{\Delta r_{i}\right\}$, and $\Delta r_{\max }=\max _{i}\left\{\Delta r_{i}\right\}$. The Radial Distance Ratio is defined as $\mathrm{RDR}=\frac{\Delta r_{\max }}{\Delta r_{\min }}$.

- Angular Distance Ratio (ADr). Let $v$ be a vertex of $G$ and let $\varrho_{v}$ be the ray passing through $v$. Let $\rho_{0}, \rho_{1}, \ldots, \rho_{h-1}(h \geq 1)$ be the distinct elements of the set $\left\{\varrho_{v} \mid v \in V\right\}$, ordered so that $\angle \rho_{0}<\angle \rho_{1}<\cdots<\angle \rho_{h-1}$. If $h>1$, define $\alpha_{i}=\left(\angle \rho_{i+1}-\angle \rho_{i}\right)$ (the indices are taken modulo $h$ and the angles are measured modulo $2 \pi), \alpha_{\min }=\min _{i}\left\{\alpha_{i}\right\}$ and $\alpha_{\max }=\max _{i}\left\{\alpha_{i}\right\}$. If $h=1$ we define $\alpha_{\min }=0$ and $\alpha_{\max }=2 \pi$. The $A n$ gular Distance Ratio is defined as $\mathrm{ADR}=\frac{\alpha_{\max }}{\alpha_{\min }}$. Notice that, when $h=1$ we have $\mathrm{ADR}=+\infty$.

We say that a radial drawing is optimal in terms of CROSSINGS if it is a planar drawing; it is optimal in terms of CURVE COMPLEXITY if it is a straightline drawing; it is optimal in terms of RADIAL DISTRIBUTION if both RDR $=1$ and $\mathrm{ADR}=1$. Ideally, one would like to produce radial drawings that satisfy the semantic requirements and are optimal in terms of all the aesthetic requirements described above. Unfortunately, this is not always possible, as showed by the next result. 
Lemma 1. There exists a planar leveled graph that does not have a radial leveled drawing optimal in terms of CROSSINGS and CURVE COMPLEXITY and RADIAL DISTRIBUTION.

Proof. Let $G=(V, E, \phi)$ be the planar leveled graph defined as follows (refer to Figure 2). $V=\left\{u_{0}, u_{1}, \ldots, u_{h-1}\right\} \cup\left\{v_{0}, v_{1}, \ldots, v_{h-1}\right\} \cup\left\{w_{0}, w_{1}, \ldots, w_{h-1}\right\}, E=$ $\left\{\left(u_{i}, u_{i+1}\right),\left(v_{i}, u_{i}\right),\left(v_{i}, u_{i+1}\right),\left(w_{i}, u_{i}\right),\left(w_{i}, u_{i+1}\right),\left(w_{i}, v_{i}\right) \mid 0 \leq i \leq h-1\right\}$ (indices are taken modulo $h), \phi\left(u_{i}\right)=0, \phi\left(v_{i}\right)=0$, and $\phi\left(w_{i}\right)=1(i=0, \ldots, h-1)$. Consider the cycle induced by vertices $\left\{u_{0}, u_{1}, \ldots, u_{h-1}\right\}$; since the drawing must be optimal in terms of CROSSINGS, each edge $\left(u_{i}, u_{i+1}\right)$ must be drawn as a chord of $C_{0}$. Vertices $v_{i}(i=0, \ldots, h-1)$ must also be drawn on $C_{0}$ and outside the polygon representing the cycle. The counterclockwise order of the vertices along $C_{0}$ must be $u_{0}, v_{0}, u_{1}, v_{1}, u_{2}, \ldots, u_{h-2}, v_{h-2}, u_{h-1}, v_{h-1}$ or the opposite one. Let $\varrho_{u_{i}}$ be the ray passing through $u_{i}(i=0, \ldots, h-1)$ and let $\alpha_{i}=\left(\angle \varrho_{u_{i+1}}-\angle \varrho_{u_{i}}\right)$. The angle $\alpha_{\min }=\min _{i}\left\{\alpha_{i}\right\}$ is at most $\frac{2 \pi}{h}$ (if we want an optimal ADR all $\alpha_{i}$ must be equal to $\left.\frac{2 \pi}{h}\right)$. Each vertex $w_{i}(i=0, \ldots, h-1)$ must be drawn inside the triangle representing the cycle $u_{i}, u_{i+1}, v_{i}$ in order to have a planar drawing, and on $C_{1}$ in order to satisfy the vertex centrality requirement. This implies that the $C_{1}$ must cross every segment $\overline{u_{i} u_{i+1}}$, i.e. the radius $r_{1}$ must be greater than the minimum distance $d_{\text {min }}$ of any of these segments from the center of the circles. The value of $d_{\min }$ is equal to $r_{0} \cos (\pi / h)$. Thus we have $r_{1}>r_{0} \cos (\pi / h)$, i.e. $\frac{r_{1}}{r_{0}}>\cos (\pi / h)$. In order to have RDR $=1$ it must be $r_{0}=2 r_{1}$, i.e. $\frac{1}{2}>\cos (\pi / h)$. This inequality is never satisfied for $h \geq 3$, and therefore, for any $h \geq 3$ it is not possible to obtain $\mathrm{RDR}=1$.

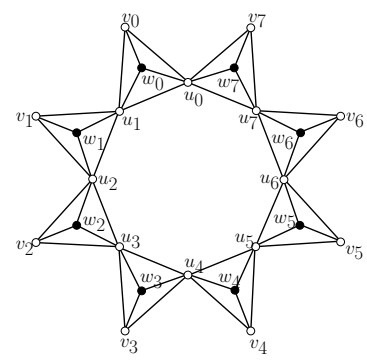

Fig. 2. A planar leveled graph that does not admit a radial leveled drawing optimal in terms of CROSSINGS and CURVE COMPLEXITY and RADIAL DISTRIBUTION. White vertices have centrality 0 ; black vertices have centrality 1 .

Lemma 1 naturally raises the question about whether one can relax one of the requirements in order to have drawings that are optimal for the other two. We consider the cases in which either CURVE COMPLEXITY or RADIAL DISTRIBUTION are relaxed. For example, it is not difficult to see that the graph used for the proof of Lemma 1 can be drawn on two circles that are sufficiently close with each other and therefore the aesthetic requirement RADIAL DISTRIBUTION is relaxed. 
However, the simultaneous optimality of CROSSINGS and CURVE COMPLEXITY cannot always be achieved. The proofs of the next two lemmas are omitted for reasons of space.

Lemma 2. There exists a planar leveled graph that does not have a planar radial leveled drawing optimal in terms of both CROSSINGS and CURVE COMPLEXITY.

Lemma 3. Every planar leveled graph with $n$ vertices has a radial leveled drawing optimal in terms of both CROSSINGS and RADIAL DISTRIBUTION, and such that CURVE COMPLEXITY is $\mathcal{O}(n)$.

Motivated by Lemma 3, we study in the next section whether one can reduce the number of bends in a planar radial drawing while maintaining optimality for RADIAL DISTRIBUTION. We will then consider trade-offs between RADIAL DISTRIBUTION and CURVE COMPLEXITY, showing how the latter can be reduced at the expenses of the former.

\section{Radial Drawings with No Crossings, Optimal Radial Distribution and Curve Complexity 3}

We describe a drawing algorithm that computes a radial drawing of a planar leveled graph $G$ with at most three bends per edge while maintaining optimal radial distribution and no crossings. An outline of our drawing technique is as follows: (i) If $G$ is not Hamiltonian, dummy edges and vertices are added so that the augmented graph is Hamiltonian and planar. (ii) The vertices of the Hamiltonian circuit are drawn as a star-shaped polygon whose vertices have the wanted centrality and have a uniform radial distribution on a polar grid. (iii) Every edge $e$ not belonging to the Hamiltonian circuit is either drawn inside or outside the polygon; if $e$ is drawn inside it has at most one bend, if it is drawn outside it has at most two bends. (iv) The dummy vertices and edges are finally removed; the construction is such that every edge can have at most three bends. Similar techniques have been previously used (see, e.g., 12 13]).

Before giving a more detailed description of the algorithm and analyzing its properties, we recall some useful definitions and results about Hamiltonian augmentation. A graph $G$ is Hamiltonian if it has a simple cycle that contains all its vertices; such a cycle is called a Hamiltonian cycle of $G$. Suppose that $G$ is planar and that $G$ is not Hamiltonian. One can augment $G$ to a (not necessarily planar) graph $G^{\prime}$, by adding to $G$ a minimal set of dummy edges such that $G^{\prime}$ contains a Hamiltonian cycle $\mathcal{H}^{\prime}$ including all the dummy edges. If $G^{\prime}$ is not planar, we can apply on $G^{\prime}$ a planarization algorithm (see, e.g., [6]) with the constraint that only crossings between dummy edges and edges of $G-\mathcal{H}^{\prime}$ are allowed. The planarization algorithm constructs an embedded planar graph $G^{\prime \prime}$ where each edge crossing is replaced with a dummy vertex. Graph $G^{\prime \prime}$ is called the augmented Hamiltonian form of $G$. If $G^{\prime}$ is planar, the augmented Hamiltonian form of $G$ is $G^{\prime}$ itself along with a given planar embedding. The vertices of the augmented Hamiltonian form that are not dummy vertices are called real 
vertices. The Hamiltonian cycle $\mathcal{H}$ of the augmented Hamiltonian form of $G$ is called an augmenting dividing Hamiltonian cycle of $G$; note that $\mathcal{H}$ is a subdivision of $\mathcal{H}^{\prime}$ obtained by possibly splitting some edges of $\mathcal{H}^{\prime}$ with dummy vertices. If every edge $e$ of $G^{\prime}$ is crossed at most $c$ times, $\mathcal{H}$ is said to be an augmenting dividing Hamiltonian cycle of $G$ with at most $c$ dummy vertices per edge. Several different techniques have been presented in the literature to compute an augmenting dividing Hamiltonian cycle of a planar graph. The following result has been proved in 8 .

Lemma 4. 8] Every planar graph $G$ with $n$ vertices admits an augmenting dividing Hamiltonian cycle $\mathcal{H}$ with at most one dummy vertex per edge. An augmented Hamiltonian form of $G$ including $\mathcal{H}$ can be computed in $O(n)$ time.

\subsection{Drawing Algorithm}

Let $G=(V, E, \phi)$ be a planar leveled graph with $n$ vertices and let $\mathcal{C}$ be a set of concentric circles such that $|\mathcal{C}|=\lambda(G)=k$ and the radius of $C_{i}$ is $r_{i}=(k-i) \cdot \Delta$, $i=0,1, \ldots, k-1$ and $\Delta>0$. This choice of the circles guarantees RDR $=1$.

Let $G^{\prime \prime}$ be an augmented Hamiltonian form of $G$ computed with the algorithm of Lemma 4 and let $\mathcal{H}$ be the Hamiltonian cycle of $G^{\prime \prime}$. Let $u_{0}$ be a vertex of $G^{\prime \prime}$ that is also a vertex of $G$; visit $\mathcal{H}$ counterclockwise starting at $u_{0}$ and let $u_{0}, u_{1}, \ldots, u_{n^{\prime \prime}-1}$ be the vertices of $G^{\prime \prime}$ in the order they are encountered during the visit. We distinguish the real vertices of $G \cap G^{\prime \prime}$ from the dummy ones by introducing a second notation for the real vertices. Vertex $u_{0}$ is also denoted $v_{0}$, vertex $v_{i}$ is the real vertex $u_{j}$ of $G \cap G^{\prime \prime}$ that is encountered after $v_{i-1}$ when visiting $\mathcal{H}$ counterclockwise.

In order to compute a drawing where $\mathrm{ADR}=1$ and the semantic requirement of vertex centrality is satisfied, we proceed as follows. Let $\rho_{i}(i=0, \ldots, n-1)$ be the ray that forms an angle of $\frac{2 \pi \cdot i}{n}$ with the positive $x$-axis. Each vertex $v_{i}$ is drawn at the intersection point $\rho_{i} \cap C_{\phi\left(v_{i}\right)}(i=0, \ldots, n-1)$.

The dummy vertices of $G^{\prime \prime}$ are drawn as follows. Let $v_{i}$ and $v_{i+1}(0 \leq i \leq n-1)$ be two vertices of $G$ such that $v_{i}=u_{j}$ and $v_{i+1}=u_{j+h}(h>1)$, i.e. two "real" vertices such that there are $h-1$ dummy vertices between them in $\mathcal{H}$. We choose $h-1$ arbitrary rays $\varrho_{1}, \varrho_{2}, \ldots, \varrho_{h-1}$ such that $\angle \rho_{i}<\angle \varrho_{1}<\angle \varrho_{2}<\cdots<\angle \varrho_{h-1}<$ $\angle \rho_{i+1}$ (for example one can choose $h-1$ equi-spaced rays). Vertex $u_{j+l}$ is drawn at point $\overline{v_{i} v_{i+1}} \cap \varrho_{l}(1 \leq l \leq h-1)$. Note that there is no semantic requirement on the dummy vertices and they do not need to be drawn on a circle of $\mathcal{C}$. The edges of $\mathcal{H}$ are drawn as straight-line segments between their endvertices. The chosen position of the vertices implies that the drawing of $\mathcal{H}$ is a star-shaped polygon, whose kernel contains the center. In the following we denote with $\mathcal{P}_{0}$ the polygon representing $\mathcal{H}$. See for example Figure 3(a)

Each edge $e$ of $G^{\prime \prime}$ not belonging to $\mathcal{H}$ is either inside or outside $\mathcal{H}$ in the planar embedding of $G^{\prime \prime}$. If $e$ is inside $\mathcal{H}$, it is drawn inside $\mathcal{P}_{0}$ as a polyline with one bend, else it is drawn outside $\mathcal{P}_{0}$ as a polyline with two bends. The edges that do not belong to $\mathcal{H}$ are suitably ordered and are inserted in the drawing one at a time in increasing order. The ordering is defined as follows. 
Let $e=\left(u_{i}, u_{j}\right)\left(0 \leq i<j \leq n^{\prime \prime}-1\right)$ be an edge that is not in $\mathcal{H}$ and let $\varrho_{u_{i}}$ and $\varrho_{u_{j}}$ be the rays through $u_{i}$ and $u_{j}$, respectively. We call span of $e$ the angle $\alpha_{e}=\min \left\{\angle \varrho_{u_{i}} \varrho_{u_{j}}, \angle \varrho_{u_{j}} \varrho_{u_{i}}\right\}$. Notice that, for each edge $e$ we have $0<\alpha_{e} \leq \pi$. We order the edges inside $\mathcal{H}$ by increasing span; similarly, the edges outside $\mathcal{H}$ are ordered by increasing span. Ties are broken arbitrarily.

DRAWING THE EDGES INSIDE $\mathcal{H}$ : Let $e_{0}, e_{2}, \ldots, e_{h-1}$ be the edges that are inside $\mathcal{H}$ ordered according to their span. The edges are drawn inside $\mathcal{P}_{0}$ so that no edge goes trough point $o$. The drawing of edge $e_{0}$ partitions polygon $\mathcal{P}_{0}$ into two sub-polygons, one of which contains $o$ and is denoted as $\mathcal{P}_{1}$. Edge $e_{l}$ is drawn inside $\mathcal{P}_{l}(l=1, \ldots, h-1)$ and partitions it into two sub-polygons one of which contains $o$ and is denoted as $\mathcal{P}_{l+1}$. Given one of the polygons $\mathcal{P}_{l}(l=0, \ldots, h-1)$, let $\mathcal{V}_{l}$ be the set of vertices (real or dummy) that are the endvertices of at least one edge $e_{g}$ with $g>l$, i.e. an edge that is not yet drawn in the drawing that defines $\mathcal{P}_{l}$. We say that $\mathcal{P}_{l}$ is weakly star-shaped, if all the vertices of $\mathcal{V}_{l}$ are visible from an internal point of $\mathcal{P}_{l}$. The set of points of $\mathcal{P}_{l}$ from which all the vertices of $\mathcal{V}_{l}$ are visible is called the weak kernel of $\mathcal{P}_{l}$ and will be denoted as $W \operatorname{Ker}\left(\mathcal{P}_{l}\right)$. When inserting edge $e_{l}$ inside polygon $\mathcal{P}_{l}$ the algorithm maintains the following invariants.

Invariant 1. All vertices of $\mathcal{V}_{l}$ are on the boundary of $\mathcal{P}_{l}$.

Invariant 2. $\mathcal{P}_{l}$ is weakly star-shaped and the weak kernel of $\mathcal{P}_{l}$ contains a closed disk centered at $o$.

We now give details about how to draw edge $e_{l}$ so that it has at most one bend and it does not go through $o$. Let $\bar{C}_{l}$ be the boundary of the closed disk contained in $W \operatorname{Ker}\left(\mathcal{P}_{l}\right)$ and let $e_{l}=\left(u_{i}, u_{j}\right)\left(0 \leq i<j \leq n^{\prime \prime}-1\right)$. Let $\varrho_{u_{i}}$ and $\varrho_{u_{j}}$ be the rays through $u_{i}$ and $u_{j}$, respectively and let $\alpha_{e_{l}}$ be the span of $e_{l}$. We have that either $\angle \varrho_{u_{i}} \varrho_{u_{j}}=\alpha_{e_{l}}$ or that $\angle \varrho_{u_{j}} \varrho_{u_{i}}=\alpha_{e_{l}}$. Assume the first case holds (the other case is analogous). If both $u_{i}$ and $u_{j}$ are real vertices, $e_{l}$ is drawn as a polyline with $u_{i}$ and $u_{j}$ as endpoints and one bend at point $b_{l}=\varrho \cap \bar{C}_{l}$, where $\varrho$ that is the bisector of the angle $\angle \varrho_{u_{i}} \varrho_{u_{j}}$. See, for example, Figure 3(b) Suppose that one of the endvertices of $e_{l}$-say $u_{i}$ - is a dummy vertex (in this case $u_{j}$ is real by Lemma 4), we draw the bend at point $b_{l}=\varrho_{u_{i}} \cap \bar{C}_{l}$. See, for example, Figure 3(c).

DrAWING THE EDGES OUTSIDE $\mathcal{H}$ : Let $e_{0}, e_{2}, \ldots, e_{h-1}$ be the edges that are outside $\mathcal{H}$ ordered according to their span. The edges are drawn outside $\mathcal{P}_{0}$ as follows. Let $\varrho_{u_{0}}, \varrho_{u_{1}}, \ldots, \varrho_{n_{n^{\prime \prime}-1}}$ be the rays passing through the points representing $u_{0}, u_{1}, \ldots, u_{n^{\prime \prime}-1}$, respectively and let $\theta_{\min }=\min _{i}\left\{\angle \varrho_{u_{i}} \varrho_{u_{i+1}}\right\}$. For every ray $\varrho_{u_{i}}$ we define two rays $\varrho_{u_{i}}^{+}$and $\varrho_{u_{i}}^{-}$; refer to Figure $3(\mathrm{~d})$. If $u_{i}$ is a real vertex $\varrho_{u_{i}}^{+}$is a ray such that $\angle \varrho_{u_{i}}^{+}=\angle \varrho_{u_{i}}+\theta_{m i n} / 3$ and $\varrho_{u_{i}}^{-}$is a ray such that $\angle \varrho_{u_{i}}^{-}=\angle \varrho_{u_{i}}-\theta_{\min } / 3$. If $u_{i}$ is a dummy vertex, $\varrho_{u_{i}}^{+}=\varrho_{u_{i}}^{-}=\varrho_{u_{i}}$.

Let $e_{l}=\left(u_{i}, u_{j}\right)$ be the current edge to be drawn $(l=0, \ldots, h-1)$. Let $\alpha_{e_{l}}$ be the span of $e_{l}$; we have that either $\angle \varrho_{u_{i}} \varrho_{u_{j}}=\alpha_{e_{l}}$ or $\angle \varrho_{u_{j}} \varrho_{u_{i}}=\alpha_{e_{l}}$. Assume the first case holds (the other case is analogous). Let $d_{\max }$ be the maximum distance from the center $o$ of the circles to any point in the drawing computed before the addition of $e_{l}$, and let $C^{*}$ be a circle centered at $o$ and having radius $r>d_{\max }$. Let $\varrho$ be the bisector of the angle $\angle \varrho_{u_{i}} \varrho_{u_{j}}$ and let $\ell_{\max }$ be the straight-line 


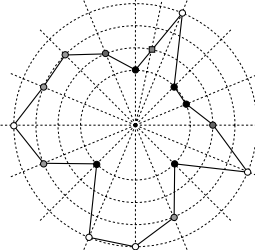

(a)

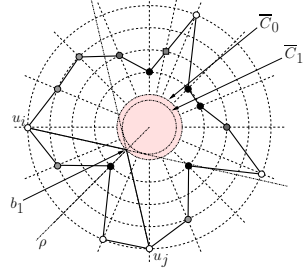

(b)

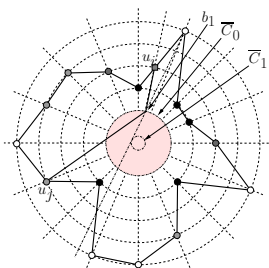

(c)

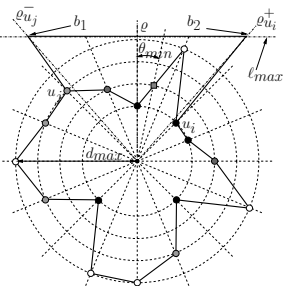

(d)

Fig. 3. Illustration of the drawing algorithm. (a) The star-shaped polygon $\mathcal{P}_{0}$ representing $\mathcal{H}$. How to draw an edge inside $\mathcal{H}$ : when both endvertices are real (b) and when an endvertex is dummy (c). (d) How to draw an edge outside $\mathcal{H}$.

orthogonal to $\varrho$ passing through the point $\varrho \cap C^{*}$. The two bends of $e_{l}$ will be drawn at points $b_{1}=\ell_{\max } \cap \varrho_{u_{i}}^{+}$and $b_{2}=\ell_{\max } \cap \varrho_{u_{j}}^{-}$. The following theorem describes the performance of the drawing algorithm both in terms of aesthetic requirements and in terms of computational complexity. The proof is omitted due to space limitation.

Theorem 1. Every planar leveled graph has a radial leveled drawing whose CURVE COMPLEXITY is 3 and that is optimal in terms of both CROSSINGS and RADiAL Distribution. Also, this drawing can be computed in $\mathcal{O}(n)$ time, where $n$ is the number of vertices of the graph.

\section{Curve Complexity and Radial Distribution: Trade-Offs}

In this section we show how to improve the quality of the drawing in terms of number of bends per edge at the expenses of a lower quality in terms of radial distribution. In Subsection 5.1 we show that at most two bends per edge can be achieved with an optimal RDR but at the price of a suboptimal ADR; Subsection 5.2 shows how to compute radial drawings with at most one bend per edge by also loosing the optimality of RDR. We recall that Lemma 2 implies that one bend per edge may be necessary if the drawing is required to be planar.

\subsection{Radial Drawings with No Crossings, Optimal Radial Distance Ratio, and Curve Complexity 2}

We describe an algorithm to compute a planar radial drawing with RDR $=1$ and CURVE COMPLEXITY 2. The algorithm computes the drawing by adding at each step a vertex and all its incident edges according to an ordering introduced by de Fraysseix, Pach and Pollack and known as a canonical ordering 5 .

Let $G$ be a maximal embedded planar graph with external boundary $u, v$, $w$. A canonical ordering of $G$ with respect to $u, v$ is an ordering of the vertices $v_{1}=u, v_{2}=v, v_{3}, \ldots, v_{n}=w$ of $G$ with the following properties for every integer 
$k$ such that $4 \leq k \leq n$ : (i) The subgraph $G_{k-1} \subseteq G$ induced by $v_{1}, v_{2}, \ldots, v_{k-1}$ is biconnected and the external boundary $B_{k-1}$ of $G_{k-1}$ contains edge $(u, v)$; (ii) $v_{k}$ is in the external face of $G_{k-1}$, and its neighbors in $G_{k-1}$ form a subpath of the path $B_{k-1}-(u, v)$.

Let $G=(V, E, \phi)$ be a planar leveled graph with $n$ vertices and let $\mathcal{C}$ be a set of concentric circles such that $|\mathcal{C}|=\lambda(G)=k$ and the radius of $C_{i}$ is $r_{i}=(k-i) \cdot \Delta$, $i=0,1, \ldots, k-1$ and $\Delta>0$. This choice of the circles guarantees RDR $=1$. Given a point $p$ in the plane $x(p)$ and $y(p)$ denote the $x$ - and $y$-coordinate of $p$, respectively. Also, we denote by $\ell_{p}$ the straight line $\{(x(p), y) \mid y \in \mathbb{R}\}$ and by $\ell_{p}^{+}$the half-line $\{(x(p), y) \mid y \geq y(p), y \in \mathbb{R}\}$.

The first step of the algorithm draws vertex $v_{1}$ at the point of coordinates $\left(-r_{\phi\left(v_{1}\right)}, 0\right)$. At the second step, $v_{2}$ is drawn at the point of coordinates $\left(r_{\phi\left(v_{2}\right)}, 0\right)$ and edge $\left(v_{1}, v_{2}\right)$ is drawn as a polyline with one bend of coordinates $(0,-y)$, where $0 \leq y \leq r_{k-1}$. At Step 3, vertex $v_{3}$ is drawn as the point of coordinates $\left(0, r_{\phi\left(v_{3}\right)}\right)$ and edges $\left(v_{1}, v_{3}\right)$ and $\left(v_{2}, v_{3}\right)$ are drawn as polylines each having one bend. The bend of $\left(v_{1}, v_{3}\right)$ has coordinates $(-x, y)$, where $0 \leq x, y \leq r_{k-1}$, while the bend of $\left(v_{2}, v_{3}\right)$ has coordinates $(x, y)$, where $0 \leq x, y \leq r_{k-1}$. At the generic Step $i(i=4, \ldots, n)$ the algorithm adds vertex $v_{i}$ to the drawing $\Gamma_{i-1}$ of the graph $G_{i-1}$ induced by vertices $v_{1}, v_{2}, \ldots, v_{i-1}$. Let $B_{i}$ be the external boundary of $G_{i}$ and let $\Pi_{i}$ be the path obtained by removing edge $\left(v_{1}, v_{2}\right)$ from $B_{i}$. The following invariants are maintained (see also Figure 4(a)):

Invariant A. Path $\Pi_{i}$ is drawn as an $x$-monotone polygonal chain in $\Gamma_{i}$.

Invariant B. Every edge $e=\left(w_{0}, w_{1}\right)$ of $\Pi_{i}$ is drawn in the half-plane $y>0$ and as a polyline with at least one bend. Let $w_{0}$ be encountered before $w_{1}$ when visiting $\Pi_{i}$ from $v_{1}$ to $v_{2}$. Let $b$ be the leftmost bend of e. The half-line $\ell_{b}^{+}$intersects all circles of $\mathcal{C}$ and does not share any point with $\Gamma_{i}$, except $b$.

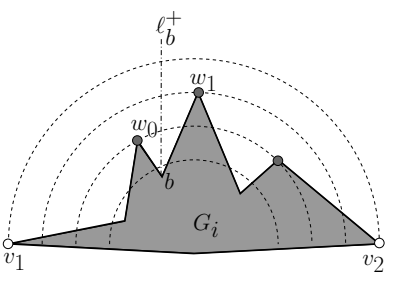

(a)

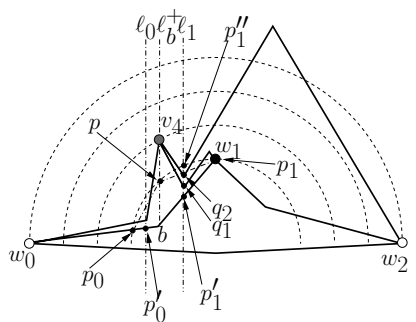

(b)

Fig. 4. Illustrations for the drawing algorithm that computes radial drawings with no crossing, optimal radial distance ratio, and curve complexity 2. (a) An example of Invariant B. (b) An example of Step $i$ of the drawing algorithm.

The addition of $v_{i}$ to $\Gamma_{i-1}$ at Step $i$ is computed as follows. Refer to Figure $4(\mathrm{~b})$. Let $w_{0}, w_{1}, \ldots, w_{h-1}$ be the vertices on $\Pi_{i-1}$ that are adjacent to $v_{i}$ and assume that they are encountered in this order when visiting $\Pi_{i-1}$ from $v_{1}$ 
to $v_{2}$. Let $b$ be the leftmost bend of $\left(w_{0}, w_{1}\right)$. Draw vertex $v_{i}$ at point $\ell_{b}^{+} \cap C_{\phi\left(v_{i}\right)}$, denote by $p_{0}$ the point $\overline{b w_{0}} \cap C_{k-1}$, by $p_{1}$ the point $\overline{b w_{1}} \cap C_{k-1}$, and by $p$ the point $\ell_{b}^{+} \cap C_{k-1}$. Let $\ell_{0}$ be any vertical straight line that intersects segment $\overline{p_{0} p}$ and let $p_{0}^{\prime}=\ell_{0} \cap\left(w_{0}, w_{1}\right)$. Edge $\left(w_{0}, v_{i}\right)$ is drawn as a polyline with one bend located at point $\left(x\left(p_{0}^{\prime}\right), y\right)$, with $y\left(p_{0}^{\prime}\right)<y<y\left(\ell_{0} \cap C_{k-1}\right)$. Let $\ell_{1}$ be any vertical straight line that intersects segment $\overline{p p_{1}}$, let $p_{1}^{\prime}=\ell_{1} \cap\left(w_{0}, w_{1}\right)$ and $p_{1}^{\prime \prime}=\ell_{1} \cap C_{k-1}$. Choose $h-1$ points, $q_{1}, q_{2}, \ldots, q_{h-1}$, on segment $\overline{p_{1}^{\prime} p_{1}^{\prime \prime}}$ with $y\left(q_{j}\right)<y\left(q_{j+1}\right)$. Each edge $\left(v_{i}, w_{j}\right)(j=1, \ldots, h-1)$ is drawn as a polyline with two bends; the first bend is at point $q_{j}$. Let $\sigma$ be the maximum value such that there exists a segment of $\Pi_{k-1}$ with slope either $\sigma$ or $-\sigma$. Choose $h-1$ positive values $\sigma_{j}(1 \leq j \leq h-1)$ such that $\sigma_{h-1}>\sigma_{h-2}>\cdots>\sigma_{1}>\sigma$. The second bend of $\left(v_{i}, w_{j}\right)$ is placed at the intersection point between the straight line with slope $\sigma_{j}$ passing trough $q_{j}$ and the straight line with slope $-\sigma_{j}$ passing trough $w_{j}(1 \leq j \leq h-1)$. The proof of the following theorem is omitted for reasons of space.

Theorem 2. Every planar leveled graph with $n$ vertices has a radial leveled drawing whose CURVE COMPLEXITY is 2 and that is optimal in terms of RDR and CROssings. Also, this drawing can be computed in $\mathcal{O}(n)$ time.

\subsection{Radial Drawings with No Crossings and Curve Complexity 1}

It has been proved that every planar graph admits a planar drawing such that all the vertices are drawn on a semi-circle and the CURVE COMPLEXITY is 1 [8]. We call a drawing with these properties a circle drawing. Let $\Gamma$ be a circle drawing and let $\overline{p_{1} q_{1}}$ and $\overline{p_{2} q_{2}}$ be two segments of $\Gamma$ that are chords of the semi-circle $C$ hosting the vertices of $\Gamma$ and such that points $p_{1}, p_{2}, q_{2}$, and $q_{1}$ are encountered in this order moving clockwise on $C$. We say that segments $\overline{p_{2} q_{2}}$ is nested inside segment $\overline{p_{1} q_{1}}$. Segments $\overline{p_{1} q_{1}}$ and $\overline{p_{2} q_{2}}$ are consecutive nested segments if $\overline{p_{2} q_{2}}$ is nested inside segment $\overline{p_{1} q_{1}}$ and there is no other segment $\overline{p_{3} q_{3}}$ such that $\overline{p_{2} q_{2}}$ is nested inside $\overline{p_{3} q_{3}}$ and $\overline{p_{3} q_{3}}$ is nested inside $\overline{p_{1} q_{1}}$. Let $\overline{p_{1} q_{1}}$ and $\overline{p_{2} q_{2}}$ be two consecutive nested segments, let $d_{a}$ be the distance from $p_{2}$ to $\overline{p_{1} q_{1}}$ and let $d_{b}$ be the distance from $q_{2}$ to $\overline{p_{1} q_{1}}$; the distance between $\overline{p_{1} q_{1}}$ and $\overline{p_{2} q_{2}}$ is the minimum value between $d_{a}$ and $d_{b}$.

To compute a planar radial drawing of a planar leveled graph $G=(V, E, \phi)$ with CURVE COMPLEXITY 1, we first construct a circle drawing $\Gamma$ by using the algorithm in [8]. Let $r$ be the radius of the semi-circle $C$ used in $\Gamma$, and let $d$ be the minimum distance between two consecutive nested segments. We choose a set of $k=\lambda(G)$ circles $C_{0}, C_{1}, \ldots, C_{k-1}$ that are concentric with $C$ and such that the radius of circle $C_{i}$ is $r_{i}=r-(i+1) \frac{\delta}{k}$, where $\delta<d$. Starting from $\Gamma$, we move each vertex $v \in V$ to the point $\varrho_{v} \cap C_{\phi(v)}$, where $\varrho_{v}$ is the ray passing through $v$, and we leave the bends at the same locations they have in $\Gamma$. The proof of the following theorem is omitted due to space limitation.

Theorem 3. Every planar leveled graph with $n$ vertices has a radial leveled drawing whose CURVE COMPLEXITY is 1 and that is optimal in terms of CROssings. Also, this drawing can be computed in $\mathcal{O}(n)$ time. 


\section{Open Problems}

We list three open problems related to the subject of this paper: (i) Based on the negative result of Lemma 1, it would be interesting to characterize the family of graphs that admit a radial leveled drawing that is optimal in terms of CROSSINGS and CURVE COMPLEXITY and RADIAL Distribution. (ii) Can one compute a radial drawing that satisfies vertex centrality, is optimal in terms of CROSSINGS and of RADiAL Distribution, and has CURVE COMPLEXity less than 3? (iii) We focused on three aesthetic requirements for radial drawings. Studying other aesthetic requirements and trade-offs can be a promising research direction.

\section{References}

1. C. Bachmaier, F. J. Brandenburg, and M. Forster. Track planarity testing and embedding. In Proc. of SOFSEM'04, volume 2, pages 3-17, 2004.

2. C. Bachmaier, F. J. Brandenburg, and M. Forster. Radial level planarity testing and embedding in linear time. JGAA, 9(1):53-97, 2005.

3. U. Brandes, P. Kenis, and D. Wagner. Communicating centrality in policy network drawings. IEEE Trans. on Vis. and Comp. Graph., 9(2):241-253, 2003.

4. U. Brandes and D. Wagner. Visone - analysis and visualization of social networks. In M. Jünger and P. Mutzel, editors, Graph Drawing Software, Springer Verlag, pages 321-340. 2004.

5. H. de Fraysseix, J. Pach, and R. Pollack. How to draw a planar graph on a grid. Combinatorica, 10:41-51, 1990.

6. G. Di Battista, P. Eades, R. Tamassia, and I. G. Tollis. Graph Drawing. Prentice Hall, 1999.

7. E. Di Giacomo, W. Didimo, G. Liotta, and H. Meijer. Computing radial drawings on the minimum number of circles. JGAA, 9(3):365-389, 2005.

8. E. Di Giacomo, W. Didimo, G. Liotta, and S. K. Wismath. Curve-constrained drawings of planar graphs. Computational Geometry, 30:1-23, 2005.

9. M. Dodge and R. Kitchin. Atlas of Cyberspace. Addison Wesley, 2001.

10. S. N. Dorogstev and J. F. F. Mendes. Evolution of Networks, From Biological Nets to the Internet and $W W W$. Oxford University Press, 2003.

11. M. Kaufmann and D. Wagner, editors. Drawing Graphs, volume 2025 of LNCS. Springer, 2001.

12. M. Kaufmann and R. Wiese. Embedding vertices at points: Few bends suffice for planar graphs. Journal of Graph Algorithms and Applications, 6(1):115-129, 2002.

13. J. Pach and R. Wenger. Embedding planar graphs at fixed vertex locations. Graph and Combinatorics, 17:717-728, 2001.

14. H. C. Purchase. Which aesthetic has the greatest effect on human understanding? In Proc. GD '97, volume 1353 of LNCS, pages 248-261. Springer, 1998.

15. H. C. Purchase. Effective information visualisation: a study of graph drawing aesthetics and algorithms. Interacting with Computers, 13(2):147-162, 2000.

16. K. Sugiyama. Graph Drawing and Applications. World Scientific, 2002.

17. R. Tamassia. Advances in the theory and practice of graph drawing. Theoretical Computer Science, 217(2):235-254, 1999.

18. R. Tamassia, G. Di Battista, and C. Batini. Automatic graph drawing and readability of diagrams. IEEE Trans. Syst., Man and Cyber., SMC-18(1):61-79, 1988. 